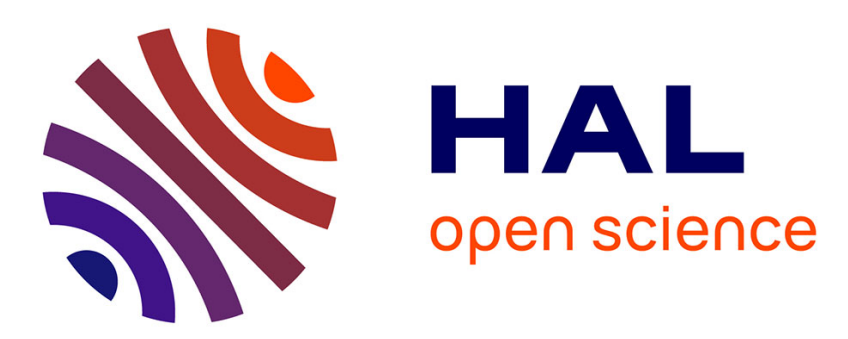

\title{
FASP juridique télévisuelle: gros plan sur les étudiants en droit et zoom arrière sur une approche pédagogique Sandrine Chapon
}

\section{To cite this version:}

Sandrine Chapon. FASP juridique télévisuelle: gros plan sur les étudiants en droit et zoom arrière sur une approche pédagogique. Recherche et Pratiques Pédagogiques en Langues de Spécialité: Cahiers de l'APLIUT, 2011, XXX (2), http://apliut.revues.org/856. hal-00911639

\section{HAL Id: hal-00911639 \\ https://hal.science/hal-00911639}

Submitted on 29 Nov 2013

HAL is a multi-disciplinary open access archive for the deposit and dissemination of scientific research documents, whether they are published or not. The documents may come from teaching and research institutions in France or abroad, or from public or private research centers.
L'archive ouverte pluridisciplinaire HAL, est destinée au dépôt et à la diffusion de documents scientifiques de niveau recherche, publiés ou non, émanant des établissements d'enseignement et de recherche français ou étrangers, des laboratoires publics ou privés. 


\section{Sandrine Chapon}

Université Pierre Mendès-France Grenoble

\section{FASP juridique télévisuelle : gros plan sur les étudiants en droit et zoom arrière sur une approche pédagogique}

Mots clés : FASP juridique, séries américaines, culture cible, anglais de spécialité, exploitation pédagogique

Résumé : Cet article présente les résultats d'une enquête de terrain menée auprès de 55 étudiants de la Faculté de droit de Grenoble en 2010. Le point de départ de l'étude est une interrogation sur la valeur ajoutée de l'utilisation des FASP télévisuelles en cours d'anglais juridique si les étudiants regardent déjà ces séries dans la sphère privée. Les résultats de l'enquête montrent que, non seulement la FASP juridique n'est pas très populaire auprès de ce public mais que, de plus, elle est regardée majoritairement en français. Ces deux éléments combinés fournissent des pistes intéressantes d'exploitation pédagogique de ce support.

\section{Legal TV FASP: A Close up Shot on Law Students and a Zoom out on a Pedagogic Approach}

Keywords: legal FASP, American TV series, target culture, English for legal purposes

Abstract: This article presents the results of a questionnaire submitted to 55 students from the Grenoble Law School in 2010. The starting point was to reflect on the benefits of using TV FASP in Legal English classes, if the students had already watched the same series for pleasure. The results of this study show that, contrary to expectations, legal FASP is not very popular amongst law students and is mainly watched in French. These combined elements provide interesting pedagogic leads in the use of legal FASP for didactic purposes. 


\title{
Sandrine Chapon*
}

\author{
Université Pierre Mendès-France Grenoble
}

\section{FASP juridique télévisuelle : gros plan sur les étudiants en droit et zoom arrière sur une approche pédagogique}

En 1999, Michel Petit a repéré des caractéristiques communes à la littérature commerciale américaine ancrée dans un milieu professionnel. Ce genre a été défini par l'appellation de «fiction à substrat professionnel » (FASP). Les séries télévisuelles juridiques relèvent aussi de cette définition. Ce sont des thrillers écrits par des avocats devenus auteurs où le héros, professionnel au service de la justice, doit affronter des ennemis au péril de sa cause, voire de sa vie.

Ces FASP ont toujours été considérées comme une valeur ajoutée dans le panel de documents «didactisables» pour l'enseignant de langue : «L'introduction du $7^{\mathrm{e}}$ art dans l'enseignement de l'anglais de spécialité juridique apporte un nouveau souffle dans une discipline réputée assez difficile d'approche » (Bourdon 2006 : 110). L'affirmation repose sur le postulat que la motivation est alimentée par le caractère novateur des supports pédagogiques. On peut lire à ce sujet les propos d'André Giordan (2005) en réponse à la question «Qu'est-ce qu'une activité désirable ? » :

Sont «désirables» des situations qui présentent de la nouveauté plutôt que de
l'habitude, donnent l'occasion de faire des choix, conduisent à des questions plutôt
qu'à des réponses ; des situations où l'individu se sent largement autonome.

Or, peut-on vraiment dire que l'utilisation pédagogique des FASP satisfait au critère de désirabilité d'une situation d'apprentissage? Les étudiants donnent l'impression de regarder déjà beaucoup de séries et ce sentiment est corroboré par les statistiques présentées par Micola et al. : 52,9\% des films diffusés sur les chaînes publiques et 60,7 \% pour les chaînes privées sont des productions américaines (2009: 5). En partant de ce constat, faut-il remettre en question l'affirmation de Muriel Bourdon ? Si les étudiants regardent pour leur plaisir les fictions proposées à l'étude en classe, où se situe le caractère innovant de la démarche ? Ne serait-il pas plutôt démotivant d'étudier un film que l'on connaît déjà ? Au vu de ces interrogations, il m'a semblé intéressant de chercher des éléments de réponse auprès des étudiants concernés par la démarche.

L'objet de cet article est donc de présenter les résultats d'une enquête de terrain menée en 2010 auprès des étudiants en droit de la faculté de Grenoble. L'objectif qui

\footnotetext{
* Sandrine Chapon, professeure certifiée, enseigne l'anglais juridique à la Faculté de Droit de Grenoble depuis 6 ans. Elle est chargée de mission aux langues et ses recherches portent sur la FASP télévisuelle comme outil didactique. 〈sandrine.chapon@upmf-grenoble.fr>.
} 
sous-tend cette démarche est de savoir ce qu'ils regardent comme séries américaines en général, et comme FASP juridique en particulier. Les données récoltées permettent d'élaborer des hypothèses concernant le type d'applications didactiques possibles. La FASP pourra être étudiée comme source lexicale et procédurale à l'intention des étudiants, mais aussi des enseignants désireux de se former en langue de spécialité. L'article montrera qu'en désaccord avec l'opinion générale des étudiants, les critiques saluent l'écriture sérielle comme genre artistique authentique.

\section{Constitution du corpus}

S'intéresser aux habitudes télévisuelles des étudiants pose le problème initial de la constitution du corpus. Se fonder sur la programmation des chaînes hertziennes n'a plus de sens depuis la généralisation du streaming ${ }^{1}$ ou du téléchargement qui ont modifié considérablement l'accessibilité des séries américaines. Bien que le téléchargement de ces œuvres soit souvent illégal, les aficionados peuvent accéder aux derniers épisodes de leurs séries préférées, moins de 48 heures après leur diffusion outre-atlantique, avec les sous-titres en prime !

Dresser une liste des séries connues des étudiants ne pouvait venir que des répondants eux-mêmes. J'ai demandé dans un premier temps à 92 étudiants de $1^{\mathrm{e}}$ année de nommer les séries américaines qu'ils connaissaient.

À partir de ces réponses, une liste de ces séries a été dressée et soumise à 55 étudiants de $3^{\mathrm{e}}$ année en leur demandant de cocher s'ils avaient regardé ces séries, et en quelle langue, et en leur laissant la possibilité d'ajouter des titres à la liste proposée. Le corpus représente un total de 55 séries (annexe 1).

La décision de procéder en deux étapes a été motivée par la nature du sujet à étudier, à savoir «la position inférieure occupée par ces dernières [les séries américaines] dans la hiérarchie culturelle » (Villez 2005 : 16). Même si les générations actuelles regardent beaucoup et aiment passionnément le genre sériel, les étudiants ont eu du mal à se départir du sentiment de culpabilité lié à l'impression «d'avouer» des goûts en inadéquation avec les attendus culturels, un sentiment bien ancré que les séries ne font pas parties des «œuvres capitales de l'humanité $»^{2}$. De ce fait, la première liste manuscrite contenait 11 séries en moyenne. Il m'a semblé plus adéquat, moins impliquant, de demander de cocher des titres pour obtenir un résultat plus proche de la réalité. Cette méthode a permis d'obtenir un écart de 6 points car, en cochant, les étudiants de $3^{\mathrm{e}}$ année ont déclaré regarder 17 séries en moyenne.

Le premier questionnaire visait à connaître les séries regardées par les étudiants afin d'observer la place occupée par le genre «FASP juridique » dans leur culture télévisuelle. Une fois le corpus des FASP juridiques déterminé, un deuxième ques-

\footnotetext{
${ }^{1}$ Lecture en mode continu d'une vidéo sur Internet.

${ }^{2}$ Une des missions du Ministère de la culture.
} 
tionnaire portant exclusivement sur leur niveau d'intérêt pour ces séries leur a été adressé pour obtenir une vision plus fine de leur comportement réel (tableau 1).

\section{Enquêtes de terrain : méthodologie mixte}

\section{Démarche quantitative}

Parmi les 55 séries, six FASP juridiques ont été nommées : Ally McBeal, The Practice, Boston Legal, Kevin Hill, Damages et Shark. Les résultats obtenus peuvent donner à penser que les étudiants en droit sont adeptes de ce genre puisque $82 \%$ des répondants mentionnent au moins une de ces séries. Mais ces résultats méritent d'être interprétés à la lumière du questionnaire concernant la fréquence à laquelle ils suivent les péripéties des hommes de loi. La question dont les résultats sont présentés ci-dessous (tableau 1), était formulée de la manière suivante : «Cochez l'affirmation qui vous semble la plus représentative de vos habitudes pour chaque série » :

\footnotetext{
Je regarde tous les épisodes ou presque de cette série (++++ dans le graphique)

Je regarde cette série assez régulièrement (+++ dans le graphique)

Je regarde cette série de manière occasionnelle (++ dans le graphique)

Je regarde cette série quand je n'ai rien d'autre à faire (+ dans le graphique)
}

Tableau 1 : Fréquence à laquelle les étudiants en droit regardent les FASP juridiques

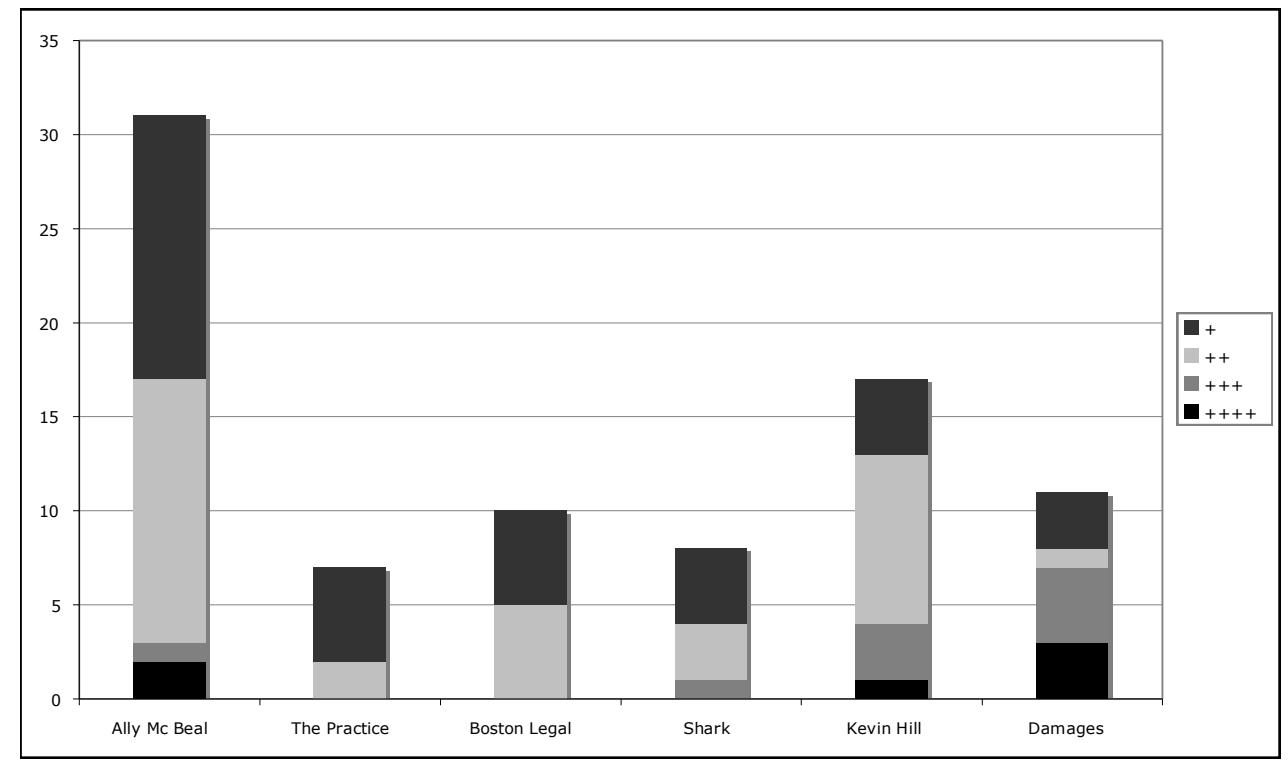

L'objectif étant d'envisager les applications pédagogiques possibles en fonction de leur connaissance des personnages et des situations filmées, il m'a semblé pertinent 
de discriminer le visionnage occasionnel, qui signifie que l'arc narratif ${ }^{3}$ est globalement connu des spectateurs/étudiants mais que le risque de connaître les enjeux d'un épisode choisi à l'étude en cours est moindre. Les étudiants ont été invités par oral à répondre qu'ils regardaient «quand ils n'ont rien d'autre à faire » uniquement quand ils ont peu ou pas de sentiment d'intimité avec les personnages fictifs.

Ainsi, les téléspectateurs d'Ally McBeal, la FASP la plus citée par les étudiants ${ }^{4}$, disent regarder cette série à $45 \%$ de manière occasionnelle et à $45 \%$ quand ils n'ont rien d'autre à faire. Il en est de même pour Kevin Hill, une des séries qui remporte le plus d'adhésion parmi les étudiants $(35 \%)$. Une seule personne la regarde de manière assidue alors que $52 \%$ la regardent occasionnellement et $23 \%$ uniquement quand ils n'ont rien d'autre à faire. Les chiffres sont encore plus éloquents quand il s'agit des séries The Practice et Boston Legal : 17 \% les regardent, mais $76 \%$ d'entre eux uniquement quand ils n'ont rien d'autre à faire.

Le seul exemple qui déroge à la tendance générale est la série Damages : si seulement $24 \%$ des étudiants qui regardent des FASP juridiques disent suivre celle-ci, $64 \%$ d'entre eux regardent tous ou presque tous les épisodes. Les raisons peuvent s'expliquer par l'originalité du scénario. Le format classique de la FASP juridique télévisuelle se caractérise par un schéma narratif indépendant à chaque épisode. Le téléspectateur peut donc regarder occasionnellement la série sans que cela nuise à sa compréhension générale. Or, contrairement aux autres séries, Damages est une série feuilletonnante, constituée d'une seule affaire de justice pour chaque saison, un recours collectif contre un chef d'entreprise accusé de délit d'initié (an insider trading class action lawsuit) pour la saison 1 et une autre action de groupe contre une entreprise polluante (an environnemental class action lawsuit) pour la saison 2. C'est donc la série dont l'arc est le plus présent puisque l'intrigue n'est résolue qu'à l'issue du dernier épisode. C'est aussi la série qui correspond le plus fidèlement à la définition de thriller puisque les avocats du cabinet Hewes and Associates ne se contentent pas de mettre leur réputation professionnelle en jeu : leur quête pour la justice menace leur vie et celle de leurs proches à chaque instant.

De manière générale, ces données révèlent que les FASP juridiques font bien partie du paysage culturel des étudiants. Par contre, s'ils sont capables d'en nommer certaines et regardent parfois un épisode, c'est de loin le genre qu'ils affectionnent le moins comparativement à d'autres.

La langue dans laquelle ils visionnent ces séries est aussi révélatrice de leur faible niveau d'intérêt. Si l'on compare les FASP juridiques à l'aune de la série dramatique la plus appréciée du public étudiant (Desperate Housewives, regardée par $73 \%$ d'entre eux), on observe que leur mode de consommation est différent. Les séries juridiques sont regardées à $86 \%$ en français alors que seulement $33 \%$ re-

\footnotetext{
${ }^{3}$ Histoire qui lie les personnages d'un épisode à l'autre (Boutet 2009 : 327).

${ }^{4} 56 \%$ de ceux qui regardent de la FASP juridique télévisuelle.
} 
gardent Desperate Housewives dans cette langue. Enfin, le fait que les étudiants ne téléchargent pas de FASP juridique démontre le faible intérêt pour le genre. Cela constitue une pratique par défaut qui relève plus d'une tentative de tuer l'ennui en zappant sur les chaînes hertziennes que d'une démarche volontaire.

Si ces chiffres permettent une lecture intéressante des habitudes télévisuelles des étudiants, leur interprétation demeure limitée. Il m'a semblé que l'enquête quantitative ne pouvait prendre tout son sens qu'en étant étayée par une démarche qualitative sur les raisons qui expliquent le faible engouement des étudiants en droit pour les FASP juridiques afin "d'élargir le répertoire des interprétations possibles de 1'expérience humaine ${ }^{5} »$ (Dörnyei citant Duff $^{6} 2007: 40$; notre traduction).

À cette fin, les étudiants ont été invités dans un premier temps, à expliciter par écrit les raisons pour lesquelles ils montrent peu d'intérêt pour ce genre et, dans un deuxième temps, à exprimer librement, lors un entretien informel, leur sentiment sur l'utilisation des films et séries en cours de langue.

\section{Démarche qualitative}

L'absence d'originalité des scénarios est le premier argument avancé par les répondants pour expliquer leur désintérêt : «C'est toujours la même chose ». De fait, les 22 épisodes de la première saison de Shark par exemple, traitent de meurtres. Il est donc vrai que ce n'est pas la singularité du synopsis qui explique sa popularité. Il en va de même pour The Practice où les affaires pénales développées (46\% des procès) sont aussi des meurtres.

Le format, qui pousse la compression temporelle à l'extrême, est presque toujours identique, non seulement d'un épisode à l'autre, mais aussi d'une série à l'autre. Le rythme est le suivant :

- une phase d'exposition du méfait commis,

- la présentation aux avocats/procureurs,

- des discussions entre professionnels du droit sur la stratégie de défense ou d'accusation à adopter,

- des scènes d'interrogatoire/contre-interrogatoire,

- le prononcé du jugement avec le suspense requis,

- le débriefing des professionnels.

La notion même de suspense ne se situe pas dans l'incertitude de l'issue des procès. Dans la série Shark, qui montre la justice du point de vue du ministère public, le procureur Stark prouve systématiquement la culpabilité des défendeurs, alors que dans la série Boston Legal, qui dépeint la perspective opposée, les leitmotive

\footnotetext{
${ }^{5}[\ldots]$ « to broaden the repertoire of possible interpretations of human experience ».

${ }^{6}$ Duff, Patricia. 2008. Case study research in applied linguistics. London: Routledge.
} 
de l'avocat septuagénaire sont « je n'ai jamais perdu un procès et n'en perdrai jamais, je suis Denny Crane » ou encore « toujours invaincu ${ }^{7} »$ (Notre traduction).

Certains étudiants mentionnent à ce titre l'absence de crédibilité des épisodes. Il est intéressant de noter à ce sujet que si le «willing suspension of disbelief » (suspension consentie de l'incrédulité) de Coleridge est inopérant dans le cas de la FASP juridique auprès des étudiants en droit, ces mêmes répondants affectionnent par ailleurs les séries policières où le professionnel résout systématiquement les énigmes en 50 minutes.

Le schéma structurel classique peut expliquer que de nombreux répondants regrettent le peu d'originalité des FASP juridiques. La place occupée par « la situation de crise » (Petit 1999:66) est très importante, laissant peu d'espace aux intrigues secondaires et cet élément est suggéré par les étudiants à travers les remarques concernant l'absence d'arc ou, comme ils le formulent, "il n'y a pas assez de sexe », «pas suffisamment d'histoires d'amour » dans la FASP juridique.

Ils expliquent aussi ne pas être attirés par le genre parce qu'ils ont « envie de voir autre chose que ce qu'ils font toute la journée ». Cette raison pourrait expliquer le très fort engouement pour les FASP médicales qui est de loin le substrat que les étudiants affectionnent le plus ${ }^{8}$. Fort de cette remarque, un questionnaire annexe concernant une éventuelle relation de réciprocité dans le comportement télévisuel des étudiants en médecine et en droit a été établi. Si les étudiants en droit regardent de la FASP médicale pour se changer les idées, est ce que les étudiants en médecine regardent de la FASP juridique pour les mêmes raisons? Un questionnaire relatif aux FASP télévisuelles juridiques et médicales a donc été distribué à 82 étudiants en médecine de $4^{\mathrm{e}}$ et $5^{\mathrm{e}}$ années de la Faculté de Dijon ${ }^{9}$ (tableaux 2 et $3)$.

Les résultats obtenus montrent que le phénomène de lassitude évoqué par les étudiants en droit n'est pas observable chez les étudiants en médecine. D'une part, ces derniers regardent encore moins les FASP juridiques que les étudiants en droit, avec seulement $17 \%$ qui s'y intéressent. D'autre part, ils semblent ne pas se reconnaître dans le besoin de se projeter dans un milieu professionnel différent de celui qu'ils côtoient habituellement. Si les étudiants en droit regardent Scrubs à $26 \%$, c’est $65 \%$ des étudiants en médecine qui déclarent suivre cette série ${ }^{10}$.

\footnotetext{
7 «Never lost, never will, Denny Crane », « still undefeated ».

${ }^{8} 96 \%$ des étudiants en droit regardent une FASP médicale et $67 \%$ regardent plus d'une série, Dr House et Grey's Anatomy étant les plus citées.

${ }^{9}$ Grâce au concours de Jean-Pierre Charpy, Maître de conférences à la Faculté de Médecine de Dijon de l'Université de Bourgogne. Ses domaines de recherche portent, entre autres, sur la FASP médicale.

${ }^{10}$ Dr House : étudiants en droit : $67 \%$, étudiants en médecine : 92,5\%. Private Practice : étudiants en droit : $1 \%$, étudiants en médecine : $27,5 \%$.
} 
Tableau 2 : Pourcentage d'étudiants par composante regardant de la FASP juridique

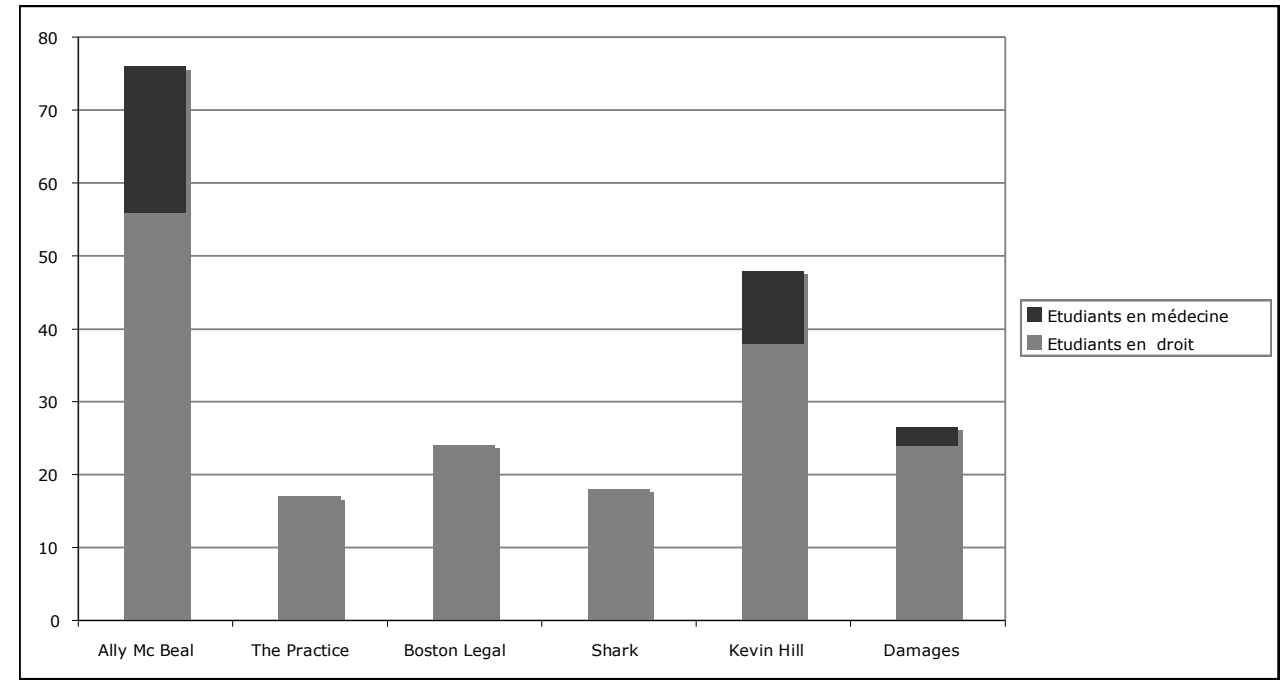

Tableau 3 : Pourcentage d'étudiants par composante regardant de la FASP médicale

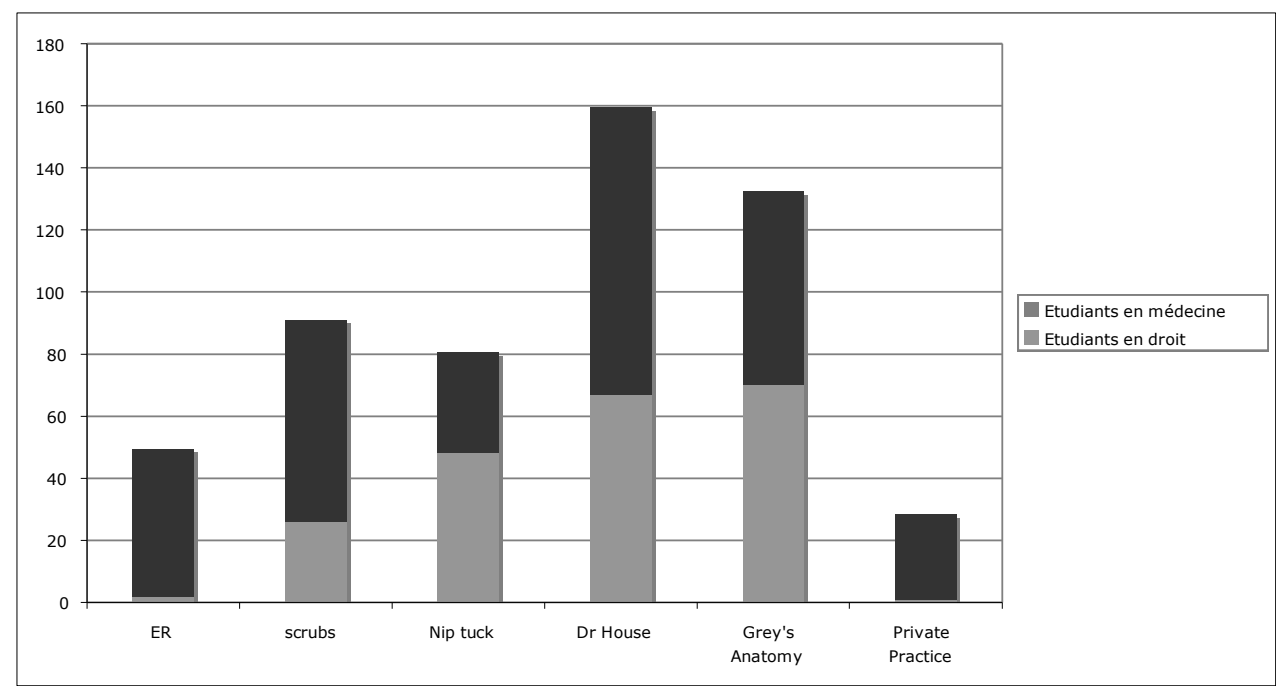

Quelle que soit l'explication avancée, scénarios peu originaux ou peu plausibles, le phénomène d'identification avec les personnages de la FASP juridique télévisuelle décrit par Villez (2005:21) semble n'opérer que faiblement sur les étudiants en droit. 


\section{Interprétation}

Plusieurs conjectures sont à explorer pour expliquer cette observation. Dans un premier temps, on peut avancer l'hypothèse que les étudiants ne s'identifient pas aux professionnels du droit parce que peu d'entre eux se destinent à la profession d'avocat. Les études de droit donnent accès à de nombreuses formations diplômantes et la possibilité d'intégrer le CRFPA ${ }^{11}$ en fait partie mais seuls $19 \%$ de la promotion de L3 de 2010 briguent cette fonction.

Une autre hypothèse repose sur le statut des spécialistes du droit portés à l'écran. Les FASP juridiques mettent en scène des professionnels. Qu'ils soient chevronnés ou débutants, c'est leur expertise qui va être la ressource servant à élucider l'intrigue. Si l'on compare ce genre aux FASP médicales, un tiers d'entre elles mettent en scène des étudiants en médecine. La trame narrative est donc constituée de problématiques professionnelles et de préoccupations estudiantines enchevêtrées les unes dans les autres et formant l'essence du thriller médical. Les contraintes de la cohabitation étudiante, les chagrins d'amour ou encore l'angoisse des examens sont des thématiques très présentes dans les FASP médicales comme Scrubs et Grey's Anatomy. Cela pourrait expliquer la proximité affective avec des personnages dont les préoccupations seraient le miroir du vécu des étudiants.

On peut aussi prendre de la distance avec les explications fournies par les étudiants qui, semble-t-il, appliquent une grille de lecture inadaptée pour juger de la qualité de la FASP juridique. En témoigne ce commentaire de Vincent Colonna (2010 : 17) : «En dehors du cinéma, la France n'a pas de cadre de pensée, de noms et de catégories, de théorie et de modèles pour aborder la fiction télévisuelle ».

Le genre sériel n'a pas vocation à représenter la réalité. Toujours selon Colonna, la série télévisuelle cultive l'art de l'hyperbole que les Américains nomment le concept du «bigger than life ${ }^{12} »(2010: 56)$. Cette amplification qui sert à produire des émotions, si elle n'est pas acceptée pour la catégorie «FASP juridique », est saluée par ailleurs, quand les étudiants parlent de façon passionnée de la série How I Met Your Mother, par exemple. Il est possible que les répondants attendent de la FASP juridique des caractéristiques relatives à la docufiction qu'ils n'exigent pas des autres séries. Ils auraient à son égard ce que Jean-Pierre Esquenazi qualifie de « conception aristocratique de la culture » $(2009: 25)$. Colonna dénonce à ce titre, la «cinéphilie hypertrophiée » des Français qui nous empêcherait, selon lui, d'apprécier la fiction télévisuelle à sa juste valeur, si on lui applique les codes du cinéma (2009: 16).

Pour revenir aux propos des étudiants, « le plaisir n'est pas tout à fait au rendezvous » et ils préfèrent d'autres séries aux FASP juridiques parce qu'ils les trouvent bien meilleures du point de vue des scénarios. Néanmoins, ils disent beaucoup

\footnotetext{
${ }^{11}$ Centre Régional de Formation Professionnelle d'Avocat.

12 «Plus grand que nature» (notre traduction).
} 
apprécier étudier des extraits de fiction cinématographique et télévisuelle en classe. Non seulement ils affirment ne pas être gênés d'étudier une FASP qu'ils connaissent déjà mais encore, ils préfèrent avoir déjà vu le film auparavant, car l'étude en cours leur apporte une lecture intéressante du fait culturel juridique (Erin Brockovich, Philadelphia, Beyond a Reasonable Doubt).

Aussi, contrairement au présupposé initial, nous avançons l'hypothèse qu'il est adéquat d'utiliser la synergie de la culture télévisuelle des étudiants comme moteur de la motivation.

\section{Pertinence de la FASP comme outil pédagogique}

\section{Pour l'apprenant}

Au vu des éléments explorés ci-dessus, un certain nombre de conclusions se dégagent. Les enquêtes de terrain révèlent que les étudiants regardent majoritairement les FASP juridiques (ainsi que les séries policières) en français ; aussi l'enseignant peut-il concevoir des scénarios pédagogiques centrés sur la traduction des termes spécialisés. Cet article n'a pas pour objet d'établir un corpus des repères culturels significatifs, mais cherche à montrer par quelques exemples d'expériences de terrain que, même si les étudiants regardent les séries juridiques ou policières, ils ne posent pas un regard distancié sur les histoires portées à l'écran et nombre d'éléments, même de nature iconographique, leur échappent.

On peut citer à ce titre le repérage du lexique relatif à la criminalistique. Le métier de la police scientifique bénéficie d'une représentation pléthorique à l'écran et ces professionnels arborent le mot «FORENSICS » en gros caractères dans le dos de leur veste mais aussi sur leur casquette et leur blouse de laboratoire, les différenciant ainsi des autres acteurs des scènes de crime. Malgré ces marqueurs identitaires visuels très apparents, aucun étudiant ne connaît le sens de ce terme.

Une approche linguistique par l'étude des traces graphiques observables à l'écran peut permettre de clarifier des concepts maintes fois entendus mais souvent assez flous chez les étudiants. Quand on leur demande ce qui est écrit dans le dos des agents de la série Les Experts Las Vegas (après en avoir montré un extrait) les réponses sont les suivantes : CSI, FBI, NCIS ou encore NYPD $(\text { sic })^{13}$, attestant, entre autres, de la confusion entre les acronymes et les notions qui s'y rapportent.

Une autre expérience, conduite auprès de 50 étudiants de première année, permet de vérifier le paradoxe soulevé par Shaeda Isani selon lequel «l'exposition à la culture professionnelle étrangère précède l'exposition à la culture professionnelle source » (2001: 117). L'auteur montre que les étudiants ont une culture juridique américaine constituée par les séries qu'ils regardent mais qu'ils ignorent que cette

\footnotetext{
${ }^{13}$ Crime Scene Investigation, Federal Bureau of Investigation, Naval Criminal Investigative Service, New York Police Department.
} 
culture n'est pas superposable à la leur.

On montre aux étudiants un extrait de film portant sur une scène de violence conjugale $^{14}$ suivie de l'arrestation de l'épouse pour «coups et blessures volontaires ayant entraîné la mort sans intention de la donner $»^{15}$. Quand on demande aux étudiants de première année de qualifier l'infraction en français, ils répondent tous sans exception que c'est un « homicide involontaire ». Or, 1'article 221-6 du code pénal français définit ce dernier comme :

Le fait de causer, dans les conditions et selon les distinctions prévues à l'article 121-3, par maladresse, imprudence, inattention, négligence ou manquement à une obligation de sécurité ou de prudence imposée par la loi ou le règlement, la mort d'autrui constitue un homicide involontaire puni de trois ans d'emprisonnement et de 45000 euros d'amende.

Cette erreur s'explique par des méprises systématiques dans la traduction du terme manslaughter lors des doublages des épisodes en langue française. Les étudiants, qui n'ont pas encore suivi les cours de procédures pénales (programme de M1), se réfèrent donc à la seule culture juridique qu'ils connaissent, c'est-à-dire celle acquise à travers les films et séries américaines.

Le dialogue entre l'épouse en garde à vue et son avocat témoigne de cette erreur récurrente de traduction. La conversation utilise les ressorts classiques de la FASP où le professionnel se livre à la définition d'un concept abscons pour ceux qui n'appartiennent pas à la communauté linguistique du droit pénal (l'épouse et le lecteur/spectateur). Le texte original est produit avec les sous-titres français officiels.

Prévenue : What does « manslaughter » mean ? (Homicide involontaire, c'est quoi ?) Avocat : It's murder without the element of intent. (C'est un crime qui n'a pas été prémédité.) ${ }^{16}$

La substitution sur l'axe paradigmatique de murder par «crime», démontre une confusion au niveau des concepts juridiques de la part du traducteur, car un crime est une catégorie d'infraction pénale (les deux autres catégories d'infractions pénales étant les délits et les contraventions, d'un degré de gravité inférieur) alors qu'un meurtre est une infraction pénale spécifique.

Par ailleurs, les assassinats, meurtres et violence volontaires ayant entrainé la mort sans intention de la donner, sont passibles de la Cour d'assises, alors qu'un homicide involontaire est un délit jugé par un tribunal correctionnel. Il est donc juridiquement inepte de qualifier un homicide involontaire de crime.

Cette erreur de traduction est particulièrement récurrente puisqu'on relève par exemple 10 épisodes sur les 22 de la première saison de Shark qui traitent de négo-

\footnotetext{
${ }^{14}$ Extrait du film The Rainmaker, 1997, adapté du roman du même nom de John Grisham.

${ }^{15}$ Article 222-7 du code pénal.

${ }^{16}$ Les sous-titres et le doublage français sont identiques.
} 
ciations d'aveux contre une requalification en «homicide involontaire». On y trouve 19 occurrences du terme manslaughter, et toutes comportent des traductions inappropriées au regard des situations dépeintes :

Seven years in Folsom for manslaughter. Beat a guy to death with a tire iron. (7 ans à Folsom pour homicide involontaire, il a battu un type à mort avec une manivelle). (Shark saison 1, épisode 15)

L'enseignant de langue peut exploiter le support FASP en vue de mettre en œuvre des procédures d'analyse critique des FASP regardées par les étudiants. La démarche vise, dans un premier temps, la prise de conscience de l'existence même du relativisme culturel qu'Edward T. Hall a appelé « la dimension cachée » de l'être humain. Les étudiants sont invités à porter un regard distancié sur ce qui leur est donné à voir. Marjolaine Boutet exprime l'aspect fonctionnel des séries en ces termes : «[Elles] permettent au spectateur averti et critique d'en savoir plus sur la société et la culture américaine à un moment donné » (2009:22). L'enseignant est à ce titre un médiateur des ajustements des modèles culturels des étudiants dans l'intention de les rendre « avertis et critiques ».

Cette démarche n'est envisageable, en revanche, que dans l'hypothèse où le concepteur de tels dispositifs pédagogiques possède les cultures qu'il va tenter de mettre en relation. Or, ce cas de figure n'est possible qu'avec l'expérience professionnelle que s'est forgée l'enseignant au fil des ans.

\section{Pour l'enseignant}

Si l'on propose une définition en creux de l'enseignant de langue de spécialité en début de carrière, il ne possède bien souvent ni les savoirs disciplinaires ni le lexique spécialisé utilisé dans la composante universitaire pour laquelle il travaille. D'aucuns se reconnaîtront dans l'expérience fastidieuse et chronophage de consulter des textes primaires dans l'objectif de se construire une compétence linguistique et culturelle qu'il va pouvoir réinvestir dans ses cours. Selon Rosalyn de Charentenay, la valeur ajoutée de la fiction par rapport au manuel de droit réside dans la dimension culturelle qui place la procédure dans un contexte (2001 : 12). Cette remarque dirigée à l'intention des étudiants est aussi valable pour les enseignants. L'objectif n'est pas de devenir un expert des champs disciplinaires; en revanche, il est indispensable d'atteindre une zone de confort qui rende la pratique professionnelle possible.

En d'autres termes, l'enseignant d'anglais de spécialité ne devrait pas devenir un enseignant des contenus disciplinaires mais plutôt un étudiant intéressé par ces contenus disciplinaires (Hutchinson et al. $1987: 163$; notre traduction) ${ }^{17}$.

Les FASP représentent à ce titre une source d'enrichissement pour l'enseignant qui va y trouver un condensé de «manifestations terminologiques et pragmatiques des

\footnotetext{
17 "In other words, the ESP teacher should not become a teacher of the subject matter, but rather an interested student of the subject matter."
} 
$-124-$

discours professionnels » (Petit 1999: 71).

Le film Jugez-moi coupable en est un parfait exemple. Inspiré par l'histoire vraie de l'affaire The United States v. Anthony Accetturo et al., une partie des dialogues des scènes du prétoire est extraite des minutes du procès qui a duré plus de 600 jours. L'acte d'accusation constitue à lui seul un document de 65 pages. Visionner le film est une alternative séduisante à la lecture de la source primaire et qui permet l'incursion dans un monde professionnel inaccessible par ailleurs.

On peut lire sous la plume de Michel Petit (1999: 74) que la FASP présente un intérêt comme support d'enseignement dans le secteur LANSAD (droit) d'une part, comme objet d'étude pour l'étudiant de LEA d'autre part, mais aussi comme objet de recherche en anglais de spécialité. A la lumière de notre expérience, nous proposons d'ajouter que la FASP est en premier lieu un moyen efficace et stimulant intellectuellement pour l'enseignant de se constituer un capital culturel, pragmatique et linguistique indispensable pour enseigner la langue de spécialité.

\section{Drôle d'En-Droit ${ }^{18}$}

Les exemples d'utilisation pédagogique présentés précédemment visent la maîtrise des codes linguistiques et procéduraux. Pour que l'ensemble des repères culturels soit cohérent et pour rendre hommage à la richesse des mondes fictionnels, il est aussi possible d'exploiter ce support comme illustration de l'art en tant que vecteur de la défense des droits fondamentaux. Le titre de cette partie, «drôle d'En-droit », reflète les préjugés de certains étudiants à l'encontre de la société américaine. Ces représentations ont la vie dure et ont été renforcées depuis les événements du 11 septembre 2001. Didactiser la FASP peut servir à lever les malentendus culturels qui consistent à confondre les décisions des gouvernements et l'opinion populaire.

La série Boston Legal, par exemple, est particulièrement représentative de la résistance de l'intelligentsia américaine face aux dénis de démocratie de l'Administration Bush. Les recours en justice d'apparence farfelus sont des prétextes à une critique acerbe du pouvoir exécutif. Le sixième épisode de la saison 4, par exemple, met en scène le pourvoi en justice des habitants de l'île de Nantucket dans le Massachusetts, qui revendiquent leur droit à posséder une bombe nucléaire (sic). Leur avocat plaide leur cause en avançant les arguments suivants :

\footnotetext{
Notre gouvernement fédéral est en faillite. [...] Notre armée est enlisée en Iraq, peut être pour les cent ans à venir, si c'est la Cour Suprême qui choisit à nouveau notre président $^{19}$. (Notre traduction)
}

Les plaidoiries d'une veine toujours incisive sont servies avec brio, notamment par l'acteur James Spader dans le rôle d'Alan Shore. Ses discours, appris par cœur par

\footnotetext{
${ }^{18}$ Site qui prolonge le cours de droit du Pr Gilles Guglielmi : <http://www.guglielmi.fr/>.

19 "Our federal government is broken. [...] Our military is bogged down in Iraq, maybe for a hundred years if the Supreme Court gets to pick our president again." (Saison 04, épisode 16).
} 
de nombreux députés démocrates (Esquenazi 2009 : 24), peuvent servir de document modèle illustrant l'art du procédé argumentatif :

\begin{abstract}
Nous devrions commencer par de petits détails comme les droits de l'homme. Je suis d'accord pour dire que l'Exécutif possède un certain talent dans ce domaine. En fait, je pense même qu'ils sont remarquables. Appeler les prisonniers de guerre des «ennemis combattants » pour éviter de respecter les conventions de Genève contre la torture, c'est tout simplement remarquable ! Baser le camp à Cuba pour ne pas être gêné par la Constitution! Remarquable ! Et selon les nouvelles directives du Pentagone c'est ma préférée et je suis sûr que vous allez aussi l'adorer - on aura bientôt des petits tribunaux à Guantanamo qui nous donneront le droit d'emprisonner à vie, voire d'exécuter les suspects sur des preuves irrecevables dans des tribunaux civils ou militaires! Imaginez pouvoir faire exécuter quelqu'un grâce à des ouï-dire ou à des aveux uniquement obtenus par la force. Remarquable ${ }^{20}$ ! (Notre traduction).
\end{abstract}

Les personnages, baroques par certains aspects, portent des valeurs humanistes. L'opinion des étudiants en droit ne rend pas justice à cette FASP qui possède à mon sens tous les ingrédients nécessaires pour être un «objet narratif réellement ambitieux fabriqué par de véritables artistes » (Esquenazi 2009 : 27).

\title{
Conclusion
}

L'hypothèse de la pertinence de la FASP comme outil didactique s'ancre dans une conception de la pédagogie des langues où les représentations initiales des étudiants sont au cœur du processus d'apprentissage. L'étude en classe permet de poser un regard nouveau sur des personnages et des scénarios familiers. Le degré d'intimité avec les personnages place les étudiants dans une sphère qui favorise l'accès au sens.

Ces enquêtes auront donc permis de mieux cerner les centres d'intérêts des étudiants afin d'ériger des ponts cognitifs entre leurs conceptions initiales et une culture cible. Elles auront permis de connaître leurs habitudes télévisuelles, ce qui possède l'avantage de proposer des thèmes en adéquation avec leurs appétences :

La seule vérité de la recherche didactique, en effet, c'est la recherche elle-même, à l'instar de la compétence fondamentale d'un enseignant, qui est d'essayer constamment de maintenir son efficacité en s'adaptant à l'évolution de ses élèves et de leur environnement. (Puren 2006 : 2)

La motivation étudiante réside dans le caractère particulièrement insolite d'inviter les personnages des séries sur les bancs de l'école. Si l'on se réfère à la définition du Robert, dictionnaire historique de la langue française, l'insolite est ce «qui

\footnotetext{
20 "We should start with the little things, like human rights. I agree that the executive branch has a particular expertise. In fact, I think they've been brilliant. Calling the prisoners enemy combatants instead of prisoners so we can end-run the Geneva Convention and torture them. Brilliant! Basing the camp in Cuba so the Constitution won't really get in our way. Brilliant. And under the Pentagon's new draft guidelines - this is my favorite, I'm sure it'll be yours - we'll have little tribunals in Guantanamo that'll allow suspects to be jailed for life or even executed on evidence that would never be admissible in civilian or military court. Imagine being able to execute somebody on triple hearsay or on a totally coerced confession. Brilliant!' (Saison 03, épisode 22).
} 
$-126-$

étonne par son caractère inaccoutumé ». Le détournement de la fonction divertissante de la télévision en fait par essence une activité curieuse. Charge au concepteur de dispositifs didactiques de conserver à l'esprit la mise en garde de Daniel Pennac: «On est prié (je vous supplie) de ne pas utiliser ces pages comme instrument de torture pédagogique $»^{21}$, en veillant à ne pas sacrifier la nature distrayante de la FASP sur l'autel de l'intérêt éducatif.

\section{Références bibliographiques}

Bourdon, M. 2006. « De l'utilisation du cinéma en anglais de spécialité « Carrières juridiques ». Les Cahiers de l'APPLIUT, vol. XXV n $1: 108-111$.

Boutet, M. 2009. Les séries télévisées pour les nuls. Paris : First Editions.

Charentenay (de), R. 2001. "La fiction à substrat professionnel (FASP) : un(e) média(tion) pas comme les autres », $A S p$, vol. 30-33: 203-213. [En ligne] http://asp.revues.org/1962 (Consult. 04 nov 2010).

Colonna, V. 2010. L'art des séries télé. Paris : Payot \& Rivages.

Dörnyei, Z. 2007. Research Methods in Applied Linguistics. Oxford University Press.

Esquenazi, J.-P. 2009. Mythologie des séries télé. Paris : Le Cavalier bleu.

Giordan, A. 2005. «Vive la motivation ». Cahiers Pédagogiques, ${ }^{\circ}$ 429-430. [En ligne] http://www.cahiers-pedagogiques.com/spip.php?article1359 (Consult. 28 mai 2010).

Isani, S. 2001. « Calques et emprunts culturels ou le paradoxe de la culture professionnelle cible comme vecteur de perceptions erronées dans la culture professionnelle source - le cinéma et les professions juridiques ». In S. Isani (dir.). 2001. Culture \& Communication en Milieu Professionnel International. Université Stendhal : CERELC, 105-120.

Hall, E. T. 1966. The Hidden Dimension. New York : Doubleday \& $\mathrm{C}^{\circ}$.

Hutchinson T. \& A. Waters. 1987. English for Specific Purposes, a learningcentred approach. Cambridge : University Press.

Micola A., A. Bris \& A. Banal-Estanol. «TV or not TV? Subtitling and English skills ». RePEc [En ligne].

$<$ http://www.econ.upf.edu/docs/papers/downloads/1156.pdf> (Consult. 16 mai 2010).

Pennac, D. 1992. Comme un roman. Saint-Amand : Gallimard.

Petit, M. 1999. «La fiction à substrat professionnel : une voie d'accès à l'anglais de spécialité ». ASp, vol. 23-26:57-81.

${ }^{21}$ Page de garde de Comme un roman.

Les Cahiers de l'APLIUT - Vol. XXX Nº 2 - JUIN 2011 - ISSN 0248-9430 
Puren, C. 2006. «Explication de textes et perspective actionnelle : la littérature entre le dire scolaire et le faire social ». [En ligne] http://langues.acdijon.fr/IMG/Article_puren_explic_textes.pdf (Consult. 27 mai 2010).

Villez, B. 2005. Séries télé, visions de la justice. Paris : PUF.

\section{Corpus des FASP juridiques}

Ally McBeal. Série créée par D. E. Kelley. Diffusée entre 1997 et 2002 sur le réseau Fox et sur M6 à partir 1998.

The Practice (titre français: The Practice: Bobby Donnell et Associés). Série créée par D. E. Kelley. Diffusée entre 1997 et le 2004 sur ABC et sur M6 à la fin des années 1990.

Boston Legal (titre français : Boston Justice). Série créée par D. E. Kelley. Diffusée entre 2004 et 2008 sur le réseau $\mathrm{ABC}$ et sur TF1 à partir 2006.

Kevin Hill. Série créée par J. A. Reyes. Diffusée entre septembre 2004 et mai 2005 sur UPN, et à partir de 2005 sur Canal+ et reprise en 2007 sur M6.

Damages. Série créée par T. A. Kessler, G. Kessler \& D. Zelman. Diffusée depuis 2007 sur FX Network et depuis 2008 sur Canal+.

Shark. Série créée par I. Biederman. Diffusée entre 2006 et 2008 sur CBS et sur M6 en 2008.

Philadelphia. 1993. Film réalisé par J. Demme.

The Rainmaker. 1997. Film réalisé par F. F. Coppola.

Erin Brokovich. 2000. Film réalisé par S. Soderbergh.

Find me guilty. 2006. Film réalisé par S. Lumet.

Beyond a Reasonable Doubt. 2009. Film réalisé par P. Hyams.

\section{Annexe : Corpus des séries américaines regardées par les étudiants en} droit de la faculté de Grenoble.

VO : Version Originale

VOSTA : Version Originale Sous Titrée en Anglais

VOSTFR : Version Originale Sous Titrée en Français

VF : Version Française

\begin{tabular}{|c|c|c|c|c|c|c|}
\hline & $\mathrm{VO}$ & $\begin{array}{c}\text { VO } \\
\text { STA }\end{array}$ & $\begin{array}{c}\text { VO } \\
\text { STFR }\end{array}$ & VF & $\begin{array}{c}\text { Total } \\
\text { oui }\end{array}$ & non \\
\hline \multicolumn{7}{|l|}{ Les FASP juridiques } \\
\hline Ally Mc Beal & 1 & 0 & 2 & 28 & 31 & 24 \\
\hline Kevin Hill & 0 & 0 & 1 & 15 & 16 & 39 \\
\hline Boston Legal (Boston justice) & 0 & 0 & 2 & 10 & 12 & 43 \\
\hline Damages & 0 & 2 & 2 & 7 & 11 & 44 \\
\hline Shark & 1 & 0 & 0 & 8 & 9 & 46 \\
\hline $\begin{array}{l}\text { The Practice (The Practice : Bobby Donnell et } \\
\text { Associés) }\end{array}$ & 0 & 0 & 1 & 6 & 7 & 48 \\
\hline
\end{tabular}

Les Cahiers de l'APLIUT - Vol. XXX N 2 - JUIN 2011 - ISSN 0248-9430 
$-128-$

\begin{tabular}{|c|c|c|c|c|c|c|}
\hline \multicolumn{7}{|l|}{ Les FASP médicales } \\
\hline Dr House & 0 & 1 & 10 & 25 & 36 & 19 \\
\hline Nip/Tuck & 2 & 0 & 6 & 18 & 26 & 29 \\
\hline Grey's Anatomy & 2 & 2 & 13 & 18 & 25 & 35 \\
\hline Scrubs & 1 & 0 & 5 & 7 & 13 & 42 \\
\hline Private Practice & 0 & 0 & 1 & 0 & 1 & 54 \\
\hline \multicolumn{7}{|l|}{ Les séries policières } \\
\hline Prison Break & 1 & 4 & 12 & 11 & 28 & 27 \\
\hline CSI Vegas, Miami, Manhattan (Les Experts) & 1 & 0 & 5 & 20 & 26 & 29 \\
\hline Cold Case (Affaires classées) & 0 & 0 & 1 & 24 & 25 & 30 \\
\hline Bones & 2 & 0 & 0 & 17 & 19 & 36 \\
\hline 24 & 1 & 2 & 5 & 9 & 17 & 38 \\
\hline Law and Order (New York Unité spéciale) & 0 & 0 & 0 & 15 & 15 & 40 \\
\hline Criminal Minds (Esprits criminels) & 0 & 0 & 1 & 8 & 9 & 46 \\
\hline Dexter & 1 & 0 & 4 & 3 & 8 & 47 \\
\hline NCIS & 1 & 0 & 1 & 5 & 7 & 48 \\
\hline The Wire (Sur écoute) & 1 & 0 & 2 & 0 & 3 & 52 \\
\hline \multicolumn{7}{|l|}{ Les séries dramatiques } \\
\hline Desperate Housewives & 2 & 6 & 20 & 14 & 42 & 13 \\
\hline Friends & 5 & 2 & 10 & 23 & 40 & 15 \\
\hline Dawson & 1 & 0 & 1 & 33 & 35 & 20 \\
\hline The Simpsons & 2 & 0 & 2 & 30 & 34 & 21 \\
\hline The Fresh Prince of Bel-Air (Le prince de Bel Air) & 2 & 0 & 0 & 27 & 29 & 26 \\
\hline Beverly Hills & 2 & 0 & 2 & 25 & 27 & 26 \\
\hline The Nanny (Une nounou d'enfer) & 1 & 0 & 0 & 25 & 26 & 29 \\
\hline One Tree Hill (Les frères Scott) & 3 & 2 & 10 & 10 & 25 & 30 \\
\hline South Park & 5 & 0 & 4 & 14 & 23 & 32 \\
\hline Melrose Place & 1 & 0 & 0 & 19 & 20 & 35 \\
\hline My Wife and Kids (Ma famille d'abord) & 0 & 0 & 0 & 19 & 19 & 36 \\
\hline Lost & 1 & 0 & 7 & 9 & 17 & 38 \\
\hline The OC (Le comté d'Orange) & 1 & 1 & 8 & 5 & 15 & 40 \\
\hline Heartbreak High (Hartley coeur à vif) & 0 & 0 & 2 & 13 & 15 & 40 \\
\hline How I Met Your Mother & 4 & 0 & 11 & 0 & 15 & 40 \\
\hline Weeds & 2 & 1 & 6 & 4 & 13 & 42 \\
\hline Californication & 2 & 1 & 7 & 2 & 12 & 43 \\
\hline Gossip Girl & 0 & 2 & 6 & 1 & 9 & 46 \\
\hline American Dad & 3 & 0 & 3 & 2 & 8 & 47 \\
\hline Six Feet Under (Six pieds sous terre) & 1 & 0 & 2 & 3 & 6 & 49 \\
\hline Two and a Half Men (Mon oncle Charlie) & 2 & 0 & 0 & 3 & 5 & 50 \\
\hline Malcom & 0 & 0 & 2 & 1 & 3 & 52 \\
\hline Brothers and Sisters & 0 & 0 & 0 & 2 & 2 & 53 \\
\hline Sex and the City & 0 & 0 & 0 & 2 & 2 & 53 \\
\hline Family Guy (Les Griffin) & 1 & 0 & 0 & 0 & 1 & 54 \\
\hline \multicolumn{7}{|l|}{ Les phénomènes paranormaux } \\
\hline Charmed & 2 & 0 & 1 & 25 & 28 & 27 \\
\hline Medium & 1 & 0 & 1 & 20 & 22 & 33 \\
\hline Smallville & 1 & 0 & 1 & 17 & 19 & 36 \\
\hline Buffy the Vampire Slayer (B. contre les vampires) & 0 & 0 & 1 & 17 & 18 & 37 \\
\hline Ghost Whisperer (Malinda entre 2 mondes) & 0 & 0 & 1 & 15 & 16 & 39 \\
\hline The Pretender (Le caméléon) & 1 & 0 & 1 & 12 & 14 & 41 \\
\hline Heroes & 0 & 0 & 5 & 6 & 11 & 44 \\
\hline Futurama & 2 & 0 & 2 & 4 & 8 & 47 \\
\hline 4400 (Les 4400) & 0 & 0 & 0 & 4 & 4 & 51 \\
\hline
\end{tabular}

